\title{
POSITIVE CENTERS AND THE BONNESEN INEQUALITY
}

\author{
MICHAEL E. GAGE
}

(Communicated by Jonathan M. Rosenberg)

\begin{abstract}
A positive center of a convex curve is a point from which the function $p(\theta) L-A-\pi p(\theta)^{2}$ is positive for all values of $\theta$. The support function is $p$ and $L$ and $A$ are the length and area of the curve, respectively. This paper proves that all convex curves have a positive center and gives an example which shows that the common centroids (Steiner point, etc.) are not necessarily positive centers. A strengthened version of the Bonnesen inequality is obtained and a simplified proof of the one-dimensional Firey inequality.
\end{abstract}

A Bonnesen-style inequality strengthens the isoperimetric inequality by implying that the isoperimetric deficit $L^{2}-4 \pi A$ of a closed plane curve $\gamma$ is greater than some positive quantity $E(\gamma)$, where $E$ can be calculated from geometric data about $\gamma$ and is zero only for circles. $L$ is the length of the curve, and $A$ represents the enclosed area.

A standard Bonnesen inequality states that what I call the Bonnesen function

$$
B(r)=r L-A-\pi r^{2}
$$

is positive for all $r \in\left[r_{\text {in }}, r_{\text {out }}\right]$, where $r_{\text {in }}$, the inradius, is the radius of one of the largest inscribed circles while the outradius $r_{\text {out }}$ is the radius of the smallest circumscribed circle. The inequality implies that the roots of $B(r)$ are real and that the interval on which $B(r)$ is positive includes $\left[r_{\text {in }}, r_{\text {out }}\right]$. From this fact and the quadratic formula for the roots of $B(r)$ it is easy to prove that

$$
L^{2}-4 \pi A \geq \pi^{2}\left(r_{\text {out }}-r_{\text {in }}\right)^{2} \text {. }
$$

Information is lost in this proof so that $(0.2)$ is strictly weaker than the inequality $(0.1)$.

Given a curve $\gamma$ that is symmetric with respect to the origin, it is also easy to show that one of the inradius circles and the outradius circle have centers at the origin. The support function with respect to the origin, $p(\theta)$, is the distance from the origin to the supporting tangent line perpendicular to the direction specified by $\theta$. The range of the support function $p$ for a symmetric curve is

Received by the editors July 17, 1989.

1980 Mathematics Subject Classification (1985 Revision). Primary 51N20, 52A40, 53A04.

Key words and phrases. Bonnesen inequality, symmetrization.

Partially supported by NSF grant DMS- 8808029 . 
exactly $\left[r_{\text {in }}, r_{\text {out }}\right]$. This means that the Bonnesen function is nonnegative for all values within the range of the support function about the point of symmetry. (See Figure 1.)

For which points in the interior of a nonsymmetric convex plane curve is it true that the range of the support function with respect to that point lies within the interval on which $B(r)$ is nonnegative? I call such points "positive centers" and this note answers this question at least in part.

The original motivation for this project comes from the proof that the inequality

$$
\frac{L A}{\pi} \geq \int p^{2} d s
$$

holds for a convex plane curve if the origin from which the support function is defined is properly chosen. This inequality is proved in $[\mathrm{G}]$ where, combined with the Schwarz inequality, it proves a one-dimensional version of Firey's conjecture; namely,

$$
\int k^{2} d s \geq \pi L / A .
$$

This in turn is used to obtain asymptotic regularity results for the curve shortening flow.

For a convex curve symmetric about a point the proof is very simple. Representing the curve by arclength $s$ we see that the map $s \rightarrow \theta(s)$ from the point to the angle $\theta$ which the normal makes with the $x$-axis is one-to-one. Choose the origin to be the point of symmetry. Hence from the above remarks it is clear that $B(p(\theta(s)))$ is positive for all $s$ and by using the formula $2 A=\int p d s$ and integrating, we get

$$
2 L A-A L-\pi \int p^{2} d s \geq 0 .
$$

For a nonsymmetric curve the inequality $(0.3)$ is proved in [G] by symmetrizing the curve about the correctly chosen point, but this is not necessary if we can pick a positive center.

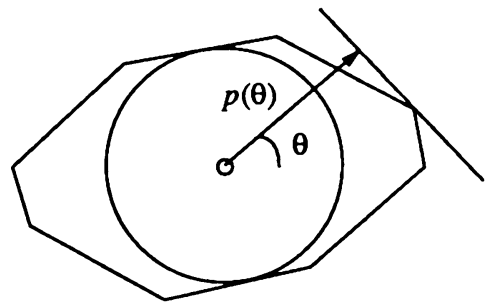

FIGURE 1 


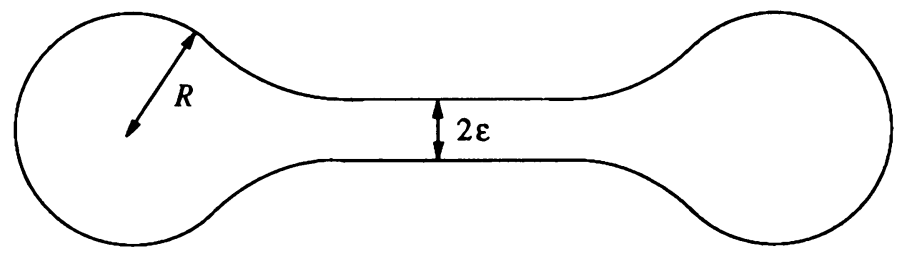

FiguRE 2

In Theorem 1.8 the existence of a positive center for any convex plane curve is proved. As a bonus we also obtain a stronger version of inequality $(0.2)$ in Theorem 1.7.

There are nonconvex symmetric curves which have no positive center. An example (due to H. Jacobowitz) is given in Figure 2. The inequality (0.4) is not satisfied on such dumbbell curves when $R$ is large relative to the thickness of the neck and the neck is very long. The existence of a positive center, on the other hand, would imply inequality $(0.3)$ which in turn would imply inequality (0.4).

It turns out that the center of the minimal annulus is always a positive center (see Theorem 1.8); however, the example in Proposition 1.11 shows that the center of mass, the centroid of the boundary, the Steiner point, and the point which minimizes the left-hand side of $(0.3)$ are in general not positive centers.

Definition 1.1. Let the inradius $r_{\text {in }}(c)$ and the outradius $r_{\text {out }}(c)$ be the radii of the largest inscribed circle centered at the point $c$ and the smallest circumscribed circle centered at $c$, respectively. Clearly $\left[r_{\text {in }}, r_{\text {out }}\right] \subseteq\left[r_{\text {in }}(c), r_{\text {out }}(c)\right]$ for any point $c$.

Definition 1.2. An inradius symmetry secant of a convex curve $\mathscr{C}$ is any secant whose inradius circle about its midpoint is tangent to $\mathscr{C}$ on each side of the secant line or is tangent at one of the points of intersection of the secant line with $\mathscr{C}$.

Definition 1.3. An inradius symmetry point is the midpoint of some inradius symmetry secant. The inradius circle about such a point is tangent to the convex curve at at least two points and lies on the midpoint of a secant line which separates the two points.

Analogous definitions define outradius symmetry secants and outradius symmetry points. A symmetry point is simultaneously an inradius symmetry point and an outradius symmetry point. Finally, as mentioned in the introduction, a point $c$ is a positive center if the range of the support function from $c$ lies within the interval on which the Bonnesen function $B(r)$ is positive.

From the standard Bonnesen inequality the following corollary holds for any centrally symmetric planar body, convex or not. The essential fact is that taking 
the convex hull tends to increase area and decrease the length of the boundary.

Corollary 1.4. The Bonnesen function of a centrally symmetric simple closed curve is positive for all $r \in\left[\tilde{r}_{\text {in }}(c), \tilde{r}_{\text {out }}(c)\right]$ where $c$ is the point of symmetry, $\tilde{r}_{\text {in }}(c)$ is the radius of the largest circle centered at $c$ inscribed in the convex hull of the original curve and $\tilde{r}_{\text {out }}$ is the outradius of the convex hull.

Proof. The length and area of a planar body are related to the length and area of its convex hull by

$$
L \geq \tilde{L}, \quad A \leq \tilde{A}
$$

where the quantities with tildes refer to the convex hull of the original body. From this it follows from $(0.1)$ that

$$
r L-A-\pi r^{2} \geq r \tilde{L}-\tilde{A}-\pi r^{2} \geq 0
$$

for all $r \in\left[\tilde{r}_{\text {in }}, \tilde{r}_{\text {out }}\right]$. This proves the corollary.

Remark 1.5. Notice that $\tilde{r}_{\text {out }}$ is equal to $r_{\text {out }}$ while, in general, $\tilde{r}_{\text {in }}>r_{\text {in }}$ if the body is not convex.

Proposition 1.6. If $c$ is an inradius symmetry point then $B\left(r_{\text {in }}(c)\right) \geq 0$. Similarly if $c$ is an outradius symmetry point then $B\left(r_{\text {out }}(c)\right) \geq 0$. If $c$ is a symmetry point then $c$ is a positive center, i.e. $B(r) \geq 0$ for all $r \in\left[r_{\text {in }}(c), r_{\text {out }}(c)\right]$.

Proof. We prove the first statement. The secant line cuts the original figure into two halves which we call the left and right halves. Let the origin be the inradius symmetry point, and form two symmetric curves about the origin, one from the left half of the original figure and one from the right half. (See Figure 3.)

The inradius of each of these symmetric curves is the same as the inradius of the original curve. In general only one of the symmetric figures will be convex.
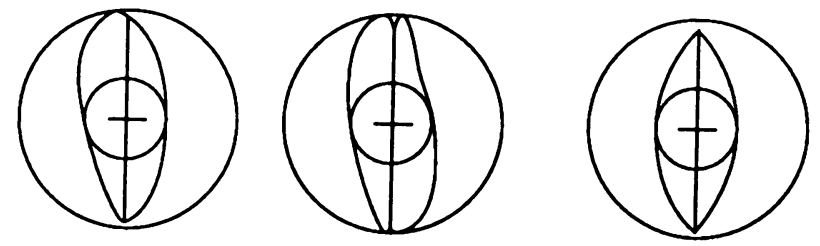

Figure 3 

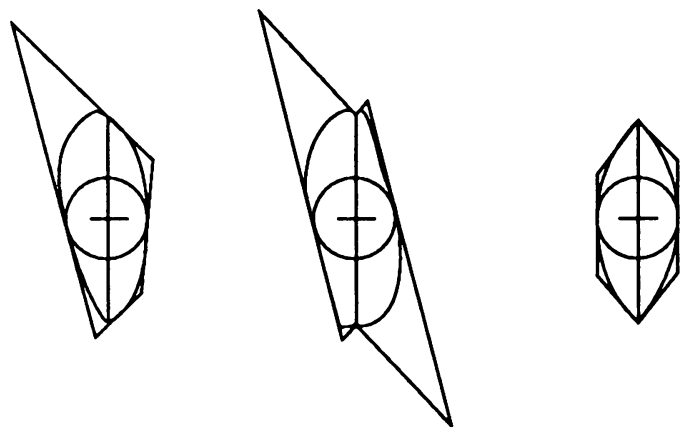

FiguRE 4

Forming the convex hull decreases the length and increases the area, and for these particular symmetric curves the inradius does not increase. To understand this last fact consider the convex quadrilateral formed by the two tangents of the curve where it touches the inradius circle and the two tangents where the curve touches the secant. Since opposite sides of this quadrilateral do not intersect it is convex. It is clear from Figure 4 that the inradius of this quadrilateral is unchanged by our symmetrization procedure, since taking the convex hull does not affect the sides tangent to the inradius circle. The original curve is contained inside the quadrilateral and, therefore, the inradius cannot increase. To complete the proof we denote the quantities pertaining to the curve formed from the left half of the original curve with subscript $l$, those pertaining to the right half with subscript $r$. Then

$$
r L^{r}-A^{r}-\pi r^{2} \geq 0 \text { for all } r \in\left[r_{\text {in }}(c), r_{\text {out }}(c)\right]
$$

and

$$
r L^{l}-A^{l}-\pi r^{2} \geq 0 \text { for all } r \in\left[r_{\text {in }}(c), r_{\text {out }}(c)\right]
$$

follow from Corollary 1.4 and the fact that the inradii and outradii about $c$ are identical with the $r_{\text {in }}(c)$ and $r_{\text {out }}(c)$ of the original curve. Since $2 L=L^{l}+L^{r}$ and $2 A=A^{l}+A^{r}$, adding these inequalities and dividing by 2 proves the result.

The proof of the second statement follows the same lines, but is easier since taking the convex hull never increases the outradius.

The third statement now follows easily since $B(r)$ is a concave function.

The strongest inequality which we obtain from these observations is

Theorem 1.7. For any closed convex curve in the plane

$$
B(r) \geq 0 \quad \text { for all } r \in\left[\min _{c} r_{\text {in }}(c), \max _{d} r_{\text {out }}(d)\right],
$$


where $c($ resp. $d)$ ranges over all inradius symmetry points (resp. outradius symmetry points) of the curve. Furthermore

$$
L^{2}-4 \pi A \geq \pi^{2}\left(\max _{d} r_{\text {out }}(d)-\min _{c} r_{\text {in }}(c)\right)^{2} .
$$

Proof. From the quadratic formula and Proposition 1.6 we have

$$
\max _{d} r_{\text {out }}(d) \leq \frac{L+\sqrt{L^{2}-4 \pi A}}{2 \pi}, \quad \min _{c} r_{\text {in }}(c) \geq \frac{L-\sqrt{L^{2}-4 \pi A}}{2 \pi} ;
$$

subtracting these inequalities and squaring proves the result.

To show that there is in fact a symmetry point (and therefore inradius symmetry points and outradius symmetry points) we prove the following theorem.

Theorem 1.8. The center $c$ of the minimal annulus is a symmetry point; hence the Bonnesen inequality (0.2) holds as does inequality (0.3).

Proof. The minimal annulus is the annulus that contains the curve and has the least width. (Since the family of annulii has finite dimension it is not difficult to show that a minimal annulus exists.) Bonnesen [B, pp. 45-51] shows that for a convex curve the minimal annulus is unique and is characterized by the following: Project the points of contact of the curve with the outer circle radially onto the inner circle. The convex hull of these projected points contains the points of contact of the curve and the inner circle. Likewise the convex hull of the points of contact with the inner circle contains the projected points. This means that there can be no line separating the points of contact along the inner circle from the points of contact along the outer circle.

From this it follows that there must be four points of contact $a, A, b, B$ (in that order along the curve) of the curve with the boundary of the annulus. The points $a$ and $b$ lie on the inner circle and the other points touch the outer circle of the annulus. To prove this, start with a point of contact $a$ on the inner circle and travel counterclockwise along the curve to the first point $A$ on the outer circle and then label $b$ the next point of contact with the inner circle. If $b$ equals $a$ then the line through $a$ tangent to the curve separates the point of contact on the inner circle, from the projections of the points of contact on the outer circle. This cannot occur for the minimal annulus. Similarly if there is no point of contact $B$ with the outer circle in traveling counterclockwise from $b$ to $a$, then the plane through $a$ and $b$ defines a closed half space which separates the points which is again a contradiction.

Now we use the intermediate value theorem to find symmetry secants through the center of the annulus. For the secant that passes through the center of the annulus and has one endpoint at $a$, the distance from $a$ to the center of the annulus is less than the distance from the center to the far end of the secant. Varying the secant so that it intersects the inner circle along the shorter arc between $a$ and $b$, we find that when the secant touches the outer circle of the annulus (at one of $A$ or $B$ ) the distance from the endpoint to the center 
exceeds the distance from the center to the opposite end. Finally, since these distances vary continuously there is an intermediate point at which the center of the annulus bisects the secant. Since we proceeded along the shorter arc of the inner circle the points $a$ and $b$ must lie on opposite sides of this secant. This proves that the center of the annulus is an inradius symmetry point.

The proof that the center is also an outradius symmetry point is analogous.

By comparing the roots of $B(r)$ with the inner and outer widths of the annulus as in Theorem 1.7 one easily proves the following corollary.

Corollary 1.9 [Bonnesen]. The inequality

$$
L^{2}-4 \pi A \geq \pi^{2}\left(r_{\text {out }}(c)-r_{\text {in }}(c)\right)^{2}
$$

holds for the minimal ("thinnest") annulus which contains the curve.

Remark 1.10. The proof of Corollary 1.9 given by Bonnesen in [B] uses a technique called annular symmetrization. This method transforms an arbitrary convex curve to a new convex curve which is symmetric about the origin and which has the same area and a boundary length which is not greater than the original. Most important the inradius and outradius remain unchanged. (Steiner symmetrization generally increases the size of the inradius, and cannot be used in proving these results.) While Bonnesen mentions only the inequality (1.5), the method of annular symmetrization can also be used to prove the inequalities in this note. Inequality (1.5) is not sharp; there is no figure for which equality holds. Bonnesen [B], using other methods, shows that the constant $\pi^{2}$ can be replaced by the sharp constant $4 \pi$ in (1.5). For many curves, however, the estimate given by the right-hand side of (1.4) will be stronger.

Among the natural centers associated with a convex curve, only the center of the minimal annulus seems to always be a positive center.

Proposition 1.11. For a convex figure, neither the center of mass, the centroid of the boundary, the Steiner point, nor the point which minimizes the left-hand side of $(0.3)$ are necessarily positive centers.

Proof. We consider the convex body in Figure 5. If $l$ is chosen large enough the lower root of the Bonnesen function can be made as close to the inradius as desired. (This is easily checked by direct calculation.) An inradius symmetry point must lie on a circle which touches two sides. The only possibilities are the horizontal midline and the angle bisectors of the two corners. The latter circles can be eliminated since there is no secant through their centers that satisfies the definition of an inradius secant.

The outradius symmetry points must be the centers of circles which are also tangent on two sides (or corners). All of the points on the vertical axis of symmetry qualify with circles which touch either the two corners or the two quarter circles. There is also a small triangle of outradius symmetry points lying near the midpoint which correspond to outradius circles that touch a corner and an adjacent quarter circle. 


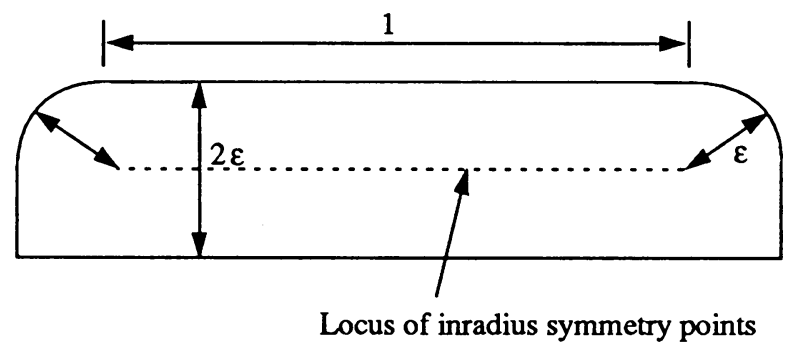

Figure 5

Examining the symmetry of the figure we see that the center of mass of the figure and the centroid of the boundary must lie on the vertical symmetry line and below the horizontal midline, and that the position is not affected by the value of $l$. Taking $l$ large gives an example of a convex body for which the center of mass and the centroid of the boundary are not positive centers. Let $X(\theta)$ be the position vector of the boundary of the figure with respect to that point. The Steiner point is given by $(2 \pi)^{-1} \int X(\theta) d \theta$. Using the same argument as above one can see that the Steiner point lies below the midline and that its position does not depend on $l$; hence it is not always a positive center. Finally the point which minimizes $\int p^{2} d s$ also satisfies $\int p N d s=0$. This point lies on the vertical symmetry line, but above the midline. The position of the point depends on $l$ in this case, but a straightforward computation shows that when $l$ is large the point lies approximately $r_{\text {in }} / 2$ above the midline.

\section{ACKNOWLEDGMENT}

I would like to thank $\mathrm{H}$. Karcher whose questions and comments encouraged me to pursue this investigation.

\section{REFERENCES}

[B] T. Bonnesen, Les problemes des isoperimetres et des isepiphanes, Gauthier-Villars et Cie, Paris, 1929.

[F] W. Firey, Shapes of worn stones, Mathematica 21 (1974), 1-11.

[G] M. Gage, An isoperimetric inequality with applications to curve shortening, Duke Math. J. 50 (1983), 1225-1229.

Department of Mathematics, University of Rochester, Rochester, New York 14627 